International Journal of Applied Mathematics

Volume 31 No. $4 \quad 2018,649-660$

ISSN: 1311-1728 (printed version); ISSN: 1314-8060 (on-line version)

doi: http://dx.doi.org/10.12732/ijam.v31i4.10

\title{
NUMERICAL INVESTIGATION OF PRESSURE HEAD JUMP \\ VALUES ON A THIN INCLUSION IN ONE-DIMENSIONAL \\ NON-LINEAR SOIL MOUSTURE TRANSPORT PROBLEM
}

Petro M. Martyniuk ${ }^{1}$, Olha R. Michuta ${ }^{2}$,

Oksana V. Ulianchuk-Martyniuk ${ }^{3}$, Mykola T. Kuzlo ${ }^{4}$

$1,2,3,4$ National University of Water

and Environmental Engineering

Rivne, UKRAINE

\begin{abstract}
A non-linear process of moisture transport in the soil containing thin inclusions has been investigated. The corresponding one-dimensional boundary value problem has been solved numerically with the finite element method. By means of numerical experiments the differences in pressure head jump values have been presented in the case of various conjugation conditions - classical and modified ones.
\end{abstract}

AMS Subject Classification: 34B60, 35Q35, 65M60

Key Words: moisture transport, porous medium, conjugation condition, pressure head jump

\section{Introduction}

The natural soils are heterogeneous on a macroscopic level. A body of porous medium includes subdomains of soils with distinct physical, chemical and mechanical properties. It concerns both natural and artificial soil bodies (e.g. hydraulic structures).

Between the layers of different soil types sometimes interfacial layers are present. In natural conditions it is especially dangerous in the context of landslides, and change of soil moisture is among the factors intensifying these processes $[1,2]$.

Received: June 5, 2018

(C) 2018 Academic Publications

$\S$ Correspondence author 
Common instances of using thin artificial inclusions of clayey soil are impervious screens in waste containment facilities. In [3] a specific type of bentonite has been discussed, which is used in geobarriers for waste containment to limit contaminant migration into the environment. As showed by the authors, the impact of bentonite membrane on contaminant transport depends on the degree of saturation.

[4] deals with geomembranes and geosynthetic clay liners used as a part of complex systems for waste utilization. Results have been presented in the form of experimental spatial and temporal redistribution of moisture in such systems under isothermal conditions.

In [5] mathematical models of moisture transport in the form of water and vapour has been developed. However, the presence of thin inclusions has not been considered there. In [6] filtration and moisture transport in saturatedunsaturated soils has been investigated. Such researches are relevant in the context of soil dams [7] and two-phase filtration processes [8].

One of the most important parts of mathematical description of moisture transport in soils containing thin inclusions is deriving conjugation conditions. These conditions are part of a boundary value problem in heterogeneous medium by which developed mathematical model is described. In works $[9,10]$ such conditions for moisture transport models has been formulated. However, unsaturated soil conductivity coefficient has been considered constant and not dependent on moisture content. Conjugation conditions for such inclusions are, generally, conditions of non-ideal contact. In $[11,12,13,14]$ theoretical problems of existence of generalized solutions in such boundary value problems have been researched.

In $[9,10]$ conjugation condition for pressure head in water flow problem has been derived assuming that pressure head $h$ is changing linearly from $h^{-}$to $h^{+}$ along the normal vector $\mathbf{n}$ to a cross-section of a thin inclusion $\gamma$ with thickness d. That means

$$
h(\zeta, t)=\frac{h^{+}-h^{-}}{d} \cdot \zeta+h^{-}, \quad 0 \leq \zeta \leq d
$$

and

$$
\frac{\partial h}{\partial \zeta}=\frac{h^{+}-h^{-}}{d} .
$$

Here $h^{+}$and $h^{-}$are pressure head values on the inclusion on $\zeta=d$ and $\zeta=$ 0 respectively. $O \zeta$ axis is tied to the normal vector of the inclusion. Then boundary conjugation condition of non-ideal contact is written as follows $[9,10]$ :

$$
\left.(\mathbf{u}, \mathbf{n})^{ \pm}\right|_{\gamma}=-k_{0} \frac{d h}{d \zeta}
$$


or

$$
\left.(\mathbf{u}, \mathbf{n})^{ \pm}\right|_{\gamma}=-\frac{k_{0}}{d}\left(h^{+}-h^{-}\right)
$$

Here $\mathbf{u}$ is water flow velocity vector. In the conditions stated above soil permeability coefficient $k_{0}$ of the thin inclusion is considered a constant value.

It is known that soil permeability coefficient (in either saturated or unsaturated conditions) depends heavily on moisture content and, thus, on pressure head $[15,16]$. For the present there are no researches concerning the influence of those dependencies on pressure head jump on a thin inclusion. Therefore above mentioned conjugation condition for mathematical models of moisture transport should be modified. Our purpose is to derive modified conditions and to evaluate their influence on the pressure head jump value.

\section{Moisture transport equation in pressure heads}

Let us consider a layer of soil with a thickness of $l$. At a depth of $x=\xi$, $0<\xi<l$ there is a thick inclusion of soil with distinct physico-mechanical and chemical characteristics. Notice that $x$ axis is oriented downwards. Then the law of moisture transport (Darcy-Klute law) becomes as follows:

$$
u=-k(h) \frac{\partial h}{\partial x}+k(h)
$$

where $u$ - water flow velocity; $h$ - pore water pressure head (in the case of saturated conditions), and suction pressure head of the soil moisture (in the case of unsaturated conditions); $k(h)$ - either saturated or unsaturated soil conductivity.

Then moisture transport equation (Richards equation) can be written as

$$
\beta(h) \frac{\partial h}{\partial t}=\frac{\partial}{\partial x}\left(k(h) \frac{\partial h}{\partial x}-k(h)\right)+f(h, x, t), x \in \Omega_{1} \bigcup \Omega_{2}, t>0,
$$

where $\Omega_{1}=(0 ; \xi), \Omega_{2}=(\xi ; l)$. Here $f(h, x, t)$ is a function comprising intensity of wetting sources and intensity of root water uptake; $\beta(h)=\frac{d \theta}{d h}$. The difference between the equation written above and the classical case is that $k(h)=\left\{k_{i}(h), x \in \Omega_{i}, i=1,2\right.$. The same concerns other coefficients and function $f$. 
652 P.M. Martyniuk, O.R. Michuta, O.V. Ulianchuk-Martyniuk, M.T. Kuzlo

\section{Nonlinear relationships between permeability coefficient and pressure head}

According to van Genuchten model $[15,16]$

$$
\begin{gathered}
\theta(h)=\theta_{\min }+\frac{\theta_{\max }-\theta_{\min }}{\left(1+(-\alpha h)^{n}\right)^{m}}, \\
\theta \in\left[\theta_{\min } ; \theta_{\max }\right], \theta_{\min }>0, \theta_{\max } \in\left(\theta_{\min } ; 1\right], n>1, m=1-\frac{1}{n} . \\
\text { Then }[15], \\
k(h)=k_{0} \sqrt{s}\left(1-\left(1-s^{\frac{1}{m}}\right)^{m}\right)^{2},
\end{gathered}
$$

where $s=\frac{\theta-\theta_{\min }}{\theta_{\max }-\theta_{\min }}-$ degree of saturation; $k_{0}$ - soil permeability (in saturated conditions with $s=1$ and $h=0)$. Further

$$
\beta(h)=\frac{d \theta}{d h}=\alpha n m\left(\theta_{\max }-\theta_{\min }\right)\left(1+(-\alpha h)^{n}\right)^{-m-1}(-\alpha h)^{n-1} .
$$

\section{Modified conjugation condition for pressure head on an inclusion}

We assume (due to the thinness of the inclusion) that moisture transport processes on a cross-section of this inclusion are stationary (or, at least, quasistationary). Thus we consider the following moisture transport problem for the inclusion:

$$
\begin{gathered}
\frac{d}{d \zeta}\left(-k^{\gamma}(h) \frac{d h}{d \zeta}+k^{\gamma}(h)\right)=0, \quad 0<\zeta<d, \\
h(0)=h^{-}, h(d)=h^{+} .
\end{gathered}
$$

Here $k^{\gamma}(h)$ is permeability of the thin inclusion that is non-linearly related to water content. From equation (1) we have

$$
k^{\gamma}(h) \frac{d h}{d \zeta}=k^{\gamma}(h)+h_{1},
$$

where $h_{1}=$ const is yet unknown constant. Further,

$$
h(\zeta)=\zeta+h_{1} \int_{0}^{\zeta} \frac{d x}{k^{\gamma}(h)}+h_{2},
$$

where $h_{2}=$ const is another yet unknown constant. Then from (3) and boundary conditions (2) we have a system of linear algebraic equations:

$$
\left\{\begin{array}{l}
h(0)=h_{2}=h^{-}, \\
h(d)=d+h_{1} \int_{0}^{d} \frac{d x}{k^{\gamma}(h)}+h_{2}=h^{+} .
\end{array}\right.
$$


From this system we have

$$
h_{2}=h^{-}, h_{1}=\frac{[h]}{\int_{0}^{d} \frac{d x}{k^{\gamma}(h)}}-\frac{d}{\int_{0}^{d} \frac{d x}{k^{\gamma}(h)}},
$$

where $[h]=h^{+}-h^{-}$is pressure head jump on the inclusion. Therefore, solution of the problem (1), (2) is a function

$$
h(\zeta)=\zeta+\int_{0}^{\zeta} \frac{d x}{k^{\gamma}(h)}\left(\frac{[h]}{\int_{0}^{d} \frac{d x}{k^{\gamma}(h)}}-\frac{d}{\int_{0}^{d} \frac{d x}{k^{\gamma}(h)}}\right)+h^{-} .
$$

This leads to

$$
\frac{d h(\zeta)}{d \zeta}=1+\frac{[h]}{k^{\gamma}(h) \int_{0}^{d} \frac{d x}{k^{\gamma}(h)}}-\frac{d}{k^{\gamma}(h) \int_{0}^{d} \frac{d x}{k^{\gamma}(h)}} .
$$

According to [9], the conjugation condition is derived based on conservation law with regard to water flux through the cross-section area unit of the surface of the inclusion along the normal vector for a small interval of time $\Delta t$. Since the flux

$$
q=\left(-k^{\gamma}(h) \frac{d h(\zeta)}{d \zeta}+k^{\gamma}(h)\right) \Delta t=u \Delta t
$$

and

$$
q=q^{+}=q^{-},
$$

then

$$
\left.u^{ \pm}\right|_{x=\xi}=-k^{\gamma}(h) \frac{d h(\zeta)}{d \zeta}+k^{\gamma}(h) .
$$

From (5) and (4) we get the final modified conjugation condition in the case of non-ideal contact for pressure head on an inclusion, permeability of which depends on the same pressure head:

$$
\left.u^{ \pm}\right|_{x=\xi}=-\frac{[h]}{\int_{0}^{d} \frac{d x}{k^{\gamma}(h)}}+\frac{d}{\int_{0}^{d} \frac{d x}{k^{\gamma}(h)}} .
$$

If $k^{\gamma}(h)=k_{0}^{\gamma}=$ const, then from the condition (6) we have the classical conjugation condition for non-ideal contact [9]. 
654 P.M. Martyniuk, O.R. Michuta, O.V. Ulianchuk-Martyniuk, M.T. Kuzlo

\section{Mathematical model for the moisture transport problem in heterogeneous soil body}

As a result, moisture transport process in heterogeneous soil body in onedimensional case is described by the following boundary value problem:

$$
\begin{gathered}
\beta(h) \frac{\partial h}{\partial t}=\frac{\partial}{\partial x}\left(k(h) \frac{\partial h}{\partial x}-k(h)\right)+f(h, x, t), x \in \Omega_{1} \bigcup \Omega_{2}, t>0 \\
\left.h(x, t)\right|_{x=l}=h_{l}(t), t \geq 0 \\
\left.u(x, t)\right|_{x=0}=Q(h, t), t \geq 0 ; \\
h(x, 0)=h_{0}(x), x \in[0 ; \xi] \bigcup[\xi ; l] ; \\
\left.u^{ \pm}\right|_{x=\xi}=-\frac{[h]}{\int_{0}^{d} \frac{d x}{k^{\gamma}(h)}}+\frac{d}{\int_{0}^{d} \frac{d x}{k^{\gamma}(h)}} .
\end{gathered}
$$

Here $\Omega_{1}=(0 ; \xi), \Omega_{2}=(\xi ; l), 0<\xi<l ; h_{l}(t), h_{0}(x), Q(h, t)$ are given functions, and the function $Q(h, t)$ comprises infiltration, evaporation and evapotranspiration. The function $h_{0}(x)$ has to be continuous on both intervals $[0 ; \xi]$ and $[\xi ; l]$. Notice that in $(7)-(11)$

$$
\begin{gathered}
k(h)=\left\{\begin{array}{ll}
k_{1}(h), & x \in \Omega_{1} ; \\
k_{2}(h), & x \in \Omega_{2} ;
\end{array} \quad \beta(h)= \begin{cases}\beta_{1}(h), & x \in \Omega_{1} ; \\
\beta_{2}(h), & x \in \Omega_{2} ;\end{cases} \right. \\
f(h, x, t)= \begin{cases}f_{1}(h, x, t), & x \in \Omega_{1} ; \\
f_{2}(h, x, t), & x \in \Omega_{2} .\end{cases}
\end{gathered}
$$

\section{Solving the moisture transport problem in a domain with inclusion using the finite element method}

As in [9], we introduce a few definitions and functional spaces. Let $H_{0}$ be a space of such functions $\varphi(x)$ which on both intervals $(0 ; \xi)$ and $(\xi ; l)$ belong to Sobolev space $W_{2}^{1}(\Omega)$, and additionally assume a non-zero value on the ends of the interval $[0, l]$, where the first type boundary conditions are set for $h(x, t)$.

Let $H$ be a space of functions $v(x, t)$ square integrable with their first derivatives $\frac{\partial v}{\partial t}, \frac{\partial v}{\partial x}$ on both intervals $(0 ; \xi),(\xi ; l), \forall t \in(0 ; T]$ and satisfy the same first type boundary conditions as $h(x, t)$. Here $T>0$. 
Multiplying equation (7) and initial condition (10) by any function $\varphi(x) \in$ $H_{0}$, integrating it over the interval $[0, l]$ and taking into consideration conjugation condition (11), we get

$$
\begin{gathered}
\int_{0}^{l} \beta(h) \frac{\partial h}{\partial t} \varphi(x) d x+\int_{0}^{l} k(h) \frac{\partial h}{\partial x} \frac{d \varphi(x)}{d x} d x+\frac{[h][\varphi]}{\int_{0}^{d} \frac{d x}{k^{\gamma}(h)}} \\
=\int_{0}^{l} f(h, x, t) \varphi(x) d x+\int_{0}^{l} k(h) \frac{d \varphi(x)}{d x} d x+\frac{d[\varphi]}{\int_{0}^{d} \frac{d x}{k^{\gamma}(h)}}, \forall t \in(0 ; T] ; \\
\int_{0}^{l} h(x, 0) \varphi(x) d x=\int_{0}^{l} h_{0}(x) \varphi(x) d x, \forall t \in(0 ; T] .
\end{gathered}
$$

Definition 1. A function $h(x, t) \in H$, that together with any $\forall \varphi(x) \in$ $H_{0}$ satisfies integral relations (12), (13) is called a generalized solution of the boundary value problem (7)-(11).

Approximate generalized solution of the boundary value problem (7)-(11) is sought in the form of

$$
h(x, t)=\sum_{i=1}^{N} a_{i}(t) \varphi_{i}(x),
$$

where $\varphi_{i}, i=\overline{2, N}$, are basis functions of a finite-dimensional subspace $M_{0} \in H_{0}$, and functions $\varphi_{1}$ are used for the purpose of approximating non-uniform first type boundary conditions in (8).

The class of functions that can be written in the form of (14) constitute space $M \subset H$.

Definition 2. An approximate generalized solution of the boundary value problem (7)-(11) is a function $\hat{h}(x, t) \in M$ that for $\forall t \in(0, T]$ and any function $\Phi(x) \in M_{0}$ satisfies integral relations

$$
\begin{gathered}
\int_{0}^{l} \beta(\hat{h}) \frac{\partial \hat{h}}{\partial t} \Phi(x) d x+\int_{0}^{l} k(\hat{h}) \frac{\partial \hat{h}}{\partial x} \frac{d \Phi(x)}{d x} d x+\frac{[\hat{h}][\Phi]}{\int_{0}^{d} \frac{d x}{k^{\gamma}(\hat{h})}} \\
=\int_{0}^{l} f(\hat{h}, x, t) \Phi(x) d x+\int_{0}^{l} k(\hat{h}) \frac{d \Phi(x)}{d x} d x+\frac{d[\Phi]}{\int_{0}^{d} \frac{d x}{k^{\gamma}(\hat{h})}}, \forall t \in(0 ; T] ; \\
\int_{0}^{l} \hat{h}(x, 0) \Phi(x) d x=\int_{0}^{l} \hat{h}_{0}(x) \Phi(x) d x, \forall t \in(0 ; T] .
\end{gathered}
$$


Solving the problem (7)-(11) with the finite element method in the form of weak formulation (12), (13) of the problem, considering (14), we have a Cauchy problem

$$
\begin{gathered}
M(A) \cdot \frac{d A}{d t}+L(A) \cdot A(t)=F(A), \\
\tilde{M} \cdot A^{(0)}=\tilde{F},
\end{gathered}
$$

where

$$
\begin{gathered}
A=\left(a_{i}(t)\right)_{i=1}^{N}, A^{(0)}=\left(a_{i}(0)\right)_{i=1}^{N}, M=\left(m_{i j}\right)_{i, j=1}^{N}, \\
L=\left(l_{i j}\right)_{i, j=1}^{N}, F=\left(f_{i}\right)_{i=1}^{N}, \tilde{F}=\left(\tilde{f}_{i}\right)_{i=1}^{N}, \tilde{M}=\left(\tilde{m}_{i j}\right)_{i, j=1}^{N}, \\
m_{i j}=\int_{0}^{l} \beta(\hat{h}) \varphi_{i} \varphi_{j} d x, \tilde{m}_{i j}=\int_{0}^{l} \varphi_{i} \varphi_{j} d x, \tilde{f}_{i}=\int_{0}^{l} h_{0} \varphi_{i} d x \\
l_{i j}=\int_{0}^{l} k(\hat{h}) \frac{d \varphi_{i}}{d x} \frac{d \varphi_{j}}{d x} d x+\frac{\left[\varphi_{i}\right]\left[\varphi_{j}\right]}{\int_{0}^{d} \frac{d x}{k^{\gamma}(\hat{h})}}, \\
f_{i}^{(1)}=\int_{0}^{l} f(\hat{h}, x, t) \varphi_{i}(x) d x+\int_{0}^{l} k(\hat{h}) \frac{d \varphi_{i}(x)}{d x} d x+\frac{d\left[\varphi_{i}\right]}{\int_{0}^{d} \frac{d x}{k^{\gamma}(\hat{h})}} .
\end{gathered}
$$

After applying temporal discretization using implicit difference scheme [17], system (15) yields

$$
M\left(A^{(p)}\right) \cdot \frac{A^{(p+1)}-A^{(p)}}{\tau}+L\left(A^{(p)}\right) \cdot A^{(p+1)}=F\left(A^{(p)}\right), p=1,2, \ldots
$$

where $\tau$ is time step, $A^{(p)}=A\left(t_{p}\right), t_{p}=p \tau$.

\section{Results of numerical experiments}

Permeability coefficient of the inclusion depends on moisture content. If in classical conjugation condition

$$
\left.u^{ \pm}\right|_{x=\xi}=-k^{\gamma} \frac{[h]}{d}+k^{\gamma}
$$

we assume $k^{\gamma}=k^{\gamma}\left(h^{-}\right)$on $x=\xi-0$ and $k^{\gamma}=k^{\gamma}\left(h^{+}\right)$on $x=\xi+0$, then letting $[h] \neq 0$ in the general case yields $u^{+} \neq u^{-}$. The latter inequality is breaking mass conservation law of the water flow through the thin inclusion. It suggests once more the necessity for modification of the conjugation conditions. 
Soil parameters for the purpose of numerical experiments are taken from the free software Hydrus-1D [16]. In particular, the basic soil is assumed to be sandy loam with $k_{0}=1.61 \mathrm{~m} /$ day, $\theta_{\min }=0.065, \theta_{\max }=0.41, n=1.89$, $\alpha=7.5$. The soil of the thin inclusion is taken as clay with the following parameters: $k_{0}^{\gamma}=0.048 \mathrm{~m} /$ day $, \theta_{\min }=0.068, \theta_{\max }=0.38, n=1.09, \alpha=0.8$.

For the model problem we consider a layer of soil with the thickness of $l=3 \mathrm{~m}$. The thin inclusion lies on the depth of $\xi=2.5 \mathrm{~m}$. Step for the $x$ coordinate is $0.01 \mathrm{~m}$. Time step $\tau=0.5$ day. Initial pressure head distribution is $h_{0}(x)=-10 \mathrm{~m}$. On the lower boundary a first type boundary condition is set with the pressure head value of $-0.1 \mathrm{~m}$.

In Table 1 volumetric moisture content jumps are given considering the gravitational term, in Table 2 - disregarding this term. Moisture content jump values while assuming $k^{\gamma}=k^{\gamma}\left(h^{+}\right)$and $k^{\gamma}=k^{\gamma}\left(h^{-}\right)$correspond to the use of classical conjugation condition, while assuming $k^{\gamma}=k^{\gamma}(h)$ - to the modified one. In parentheses the relative increase or decrease of the moisture content jump with respect to the cases of classical and modified conjugation conditions is given. In Table 1 these relative differences range from $-8.1 \%$ to $+6.6 \%$, while in Table 2 - from $-34.7 \%$ to $34.7 \%$. Moreover, the amplitude of differences increases with the increment of inclusion thickness.

\begin{tabular}{|l|l|l|l|}
\hline $\begin{array}{l}\text { Thickness of } \\
\text { the inclusion } \\
\text { and time } \\
\text { interval }\end{array}$ & $\begin{array}{l}{[\theta] \quad \text { (assuming }} \\
\left.k^{\gamma}=k^{\gamma}\left(h^{+}\right)\right)\end{array}$ & $\begin{array}{l}{[\theta] \quad \text { assuming }} \\
\left.k^{\gamma}=k^{\gamma}\left(h^{-}\right)\right)\end{array}$ & $\begin{array}{l}{[\theta] \quad \text { (assuming }} \\
\left.k^{\gamma}=k^{\gamma}(h)\right)\end{array}$ \\
\hline $\begin{array}{l}d=0.3 m \\
t=50 \text { days }\end{array}$ & $\begin{array}{l}0.0277 \\
(+6.6 \%)\end{array}$ & $\begin{array}{l}0.0320 \\
(-8.1 \%)\end{array}$ & 0.0296 \\
\hline$d=0.3 m$ & 0.0263 & 0.0272 & 0.0267 \\
$t=350$ days & $(+1.4 \%)$ & $(-1.7 \%)$ & \\
\hline$d=0.1 m$ & 0.0125 & 0.0136 & 0.0130 \\
$t=50$ days & $(+4.3 \%)$ & $(-4.8 \%)$ & \\
\hline$d=0.1 m$ & 0.0112 & 0.0114 & 0.0113 \\
$t=350$ days & $(+0.7 \%)$ & $(-0.8 \%)$ & \\
\hline
\end{tabular}

Table 1. Volumetric moisture content jump values gravitational term taken into account 


\begin{tabular}{|l|l|l|l|}
\hline $\begin{array}{l}\text { Thickness of } \\
\text { the inclusion } \\
\text { and time } \\
\text { interval }\end{array}$ & $\begin{array}{l}{[\theta] \quad \text { (assuming }} \\
\left.k^{\gamma}=k^{\gamma}\left(h^{+}\right)\right)\end{array}$ & $\begin{array}{l}{[\theta] \quad \text { (assuming }} \\
\left.k^{\gamma}=k^{\gamma}\left(h^{-}\right)\right)\end{array}$ & $\begin{array}{l}{[\theta] \quad \text { (assuming }} \\
\left.k^{\gamma}=k^{\gamma}(h)\right)\end{array}$ \\
\hline$d=0.3 m$ & 0.0737 & 0.1503 & \\
$t=50$ days & $(+33.9 \%)$ & $(-34.7 \%)$ & 0.1115 \\
\hline$d=0.3 m$ & 0.0598 & 0.1109 & 0.0916 \\
$t=350$ days & $(+34.7 \%)$ & $(-21.0 \%)$ & \\
\hline$d=0.1 m$ & 0.0298 & 0.0392 & 0.0332 \\
$t=50$ days & $(+10.5 \%)$ & $(-18.0 \%)$ & \\
\hline$d=0.1 m$ & 0.0090 & 0.0138 & 0.0108 \\
$t=350$ days & $(+16.9 \%)$ & $(-26.9 \%)$ & \\
\hline
\end{tabular}

Table 2. Volumetric moisture content jump values disregarding the gravitational term

The results of solving the model problem show that the functional relationship between permeability coefficient of thin low-permeable inclusions and moisture content (suction pressure) cannot be neglected. Besides, the moisture content jump can be influenced by regarding or disregarding the gravitational term of water flow. To conduct an assessment on real geological formations, soil parameters of all subdomains must be known, as well as the inclusion itself.

\section{Conclusions}

In this paper the process of moisture transport in the soil containing thin inclusions has been numerically investigated. The purpose is to find out a relation between pressure head jump and permeability coefficient of the inclusion, that is, in its own turn, dependent on the pressure head. Discontinuous generalized solution of corresponding boundary value problem has been found using the finite element method and numerical experiments have been conducted. In the following papers formulating a more general setting of the problem and considering two and three-dimensional cases are planned, as well as conducting theoretical investigations of quality characteristics of the found approximate solution. 


\section{References}

[1] L. Di Matteo, C. Pauselli, D. Valigi, M. Ercoli, M. Rossi, G. Guerra, C. Cambi, R. Ricco, G. Vinti, Reliability of water content estimation by profile probe and its effect on slope stability, Landslides, 15, No 1 (2018), 173-180.

[2] D.-J. Wang, H.-M. Tang, Y.-H. Zhang, C.-D. Li, L. Huang, An improved approach for evaluating the time-dependent stability of colluvial landslides during intense rainfall, Environmental Earth Sciences, 76 (2017), 321.

[3] K.M. Sample-Lord, C.D. Shackelford, Membrane behavior for unsaturated bentonite barriers, In: Geo-Congress 2014 (2014), 1900-1909.

[4] F.M. Azad, D.W. Airey, R.K. Rowe, A. El-Zein, Laboratory investigation of moisture content redistribution on the base of double composite liner systems, In: 6th International Congress on Environmental Geotechnics, New Delhi, India (2010), 354-359.

[5] P.M. Martyniuk, M.T. Kuzlo, S.K. Matus, T.P. Tsvietkova, Mathematical model of nonisothermal moisture transference in the form of water and vapor in soils in the case of chemical internal erosion, Far East J. of Mathematical Sciences, 102, No 12 (2017), 3211-3221.

[6] A.P. Vlasyuk, T.P. Tsvetkova, Mathematical simulation of the transport of salt in the case of filtration and moisture transfer in saturated-unsaturated soils in a moistening regime, J. of Engineering Physics and Thermophysics, 88, No 5 (2015), 1062-1073.

[7] N. Ivanchuk, P. Martynyuk, T. Tsvetkova, O. Michuta, Mathematical modeling and computer simulation of the filtration processes in earth dams, Eastern-European J. of Enterprise Technologies, 86, No 2/6 (2017), 63-69.

[8] A.Ya. Bomba, S.V. Yaroshchak, Complex approach to modeling of twophase filtration processes under control conditions, J. of Mathematical Sciences, 184, No 1 (2012), 56-68.

[9] I.V. Sergienko, V.V. Skopetskyi, V.S. Deineka, Mathematical Modeling and Research of Processes in Heterogeneous Environments, Naukova Dumka, Kiev (1991) (in Russian).

[10] I.V. Sergienko, V.S. Deineka, Optimal Control of Distributed Systems With Conjugation Conditions, Kluwer Academic Publishers, New York (2005). 
660 P.M. Martyniuk, O.R. Michuta, O.V. Ulianchuk-Martyniuk, M.T. Kuzlo

[11] S.I. Lyashko, D.A. Nomirovskii, The generalized solvability and optimization of parabolic systems in domains with thin low-permeable inclusions, Cybernetics and Systems Analysis, 39, No 5 (2003), 737-745.

[12] D.A. Nomirovskii, Generalized solvability of parabolic systems with nonhomogeneous transmission conditions of nonideal contact type, Differential Equations, 40, No 10 (2004), 1467-1477.

[13] D.A. Nomirovskii, Generalized solvability and optimization of a parabolic system with a discontinuous solution, J. of Differential Equations, 233, No 1 (2007), 1-21.

[14] D.A. Nomirovskii, O.I. Vostrikov, Generalized statements and properties of models of transport processes in domains with cuts, Cybernetics and Systems Analysis, 52, No 6 (2016), 931-942.

[15] M.T. van Genuchten, A closed-form equation for predicting the hydraulic conductivity of unsaturated soils, Soil Sci. Soc. Am. J., 44, No 5 (1980), 892-898.

[16] J. Simunek, M.T. van Genuchten, M. Sejna, Recent developments and applications of the HYDRUS computer software packages, Vadose Zone J., 15 (2016), 1-25.

[17] V. Herus, O. Stepanchenko, P. Martyniuk, O. Michuta, Two-dimensional problem concerning contact suffusion interplay on the filtration consolidation processes of heterogeneous soils, International J. of Pure and Appl. Math., 11, No 2 (2017), 407-418. 